\title{
A NEW VARIABLE EXPONENT PICONE IDENTITY AND APPLICTIONS
}

\author{
TINGFU FENG AND JUNQIANG HAN
}

\begin{abstract}
In this paper, we derive a new variable exponent Picone identity for $p(x)$-Laplacian, which contains some known Picone identities. As applications, a strict monotonicity of principal eigenvalues with respect to domains for the eigenvalue problems to $p(x)$-Laplace equation, a variable exponent Barta type inequality, a variable exponent Hardy type inequality with weight, a Sturmian comparison principle to $p(x)$-Laplace equation and a Liouville type theorem to $p(x)$ Laplace system are shown.
\end{abstract}

Mathematics subject classification (2010): 26D10, 35J25.

Keywords and phrases: Variable exponent Picone identity, $p(x)$-Laplacian; principal eigenvalue, Sturmian comparison principle, Liouville type theorem.

\section{REFERENCES}

[1] W. Allegretto, Positive solutions and spectral properties of weakly coupled elliptic systems, J. Math. Anal. Appl. 120, 2 (1986), 723-729.

[2] W. Allegretto And Y. X. Huang, A Picone's identity for the p-laplacian and applications, Nonlinear Anal. 32, 7 (1998), 819-830.

[3] W. Allegretto, Form estimates for the $p(x)$-Laplacean, Proc. Amer. Math. Soc. 135, 7 (2007), $2177-2185$.

[4] E. Acerbi, G. Mingione And G. A. Seregin, Regularity results for parabolic systems related to a class of non-Newtonian fluids, Ann. Inst. H. Poincaré Anal. Non Linéaire 21, 1 (2004), 25-60.

[5] S. N. Antontsev and J. F. Rodrigues, On stationary thermo-rheological viscous flows, Ann. Univ. Ferrara Sez. VII Sci. Mat. 52, 1 (2006), 19-36.

[6] R. Aboulaich, D. Meskine And A. Souissi, New diffusion models in image processing, Comput. Math. Appl. 56, 4 (2008), 874-882.

[7] C. O. ALVES, Existence of solution for a degenerate $p(x)$-Laplacian equation in $R^{N}$, J. Math. Anal. Appl. 345, 2 (2008), 731-742.

[8] E. M. Bollt, R. Chartrand And S. Esedoglu, ET AL., Graduated adaptive image denoising: local compromise between total variation and isotropic diffusion, Adv. Comput. Math. 31, 1 (2009), $61-85$.

[9] K. BAL, Generalized Picone's identity and its applications, Electron. J. Differential Equations 2013, 243 (2013), 1-6.

[10] Y. CHEN, S. LEVINE AND M. RAO, Variable exponent, linear growth functionals in image restoration, SIAM J. Appl. Math. 66, 4 (2006), 1383-1406.

[11] L. Diening, P. HÄSTÖ AND S. RoudEnKo, Function spaces of variable smoothness and integrability, J. Funct. Anal. 256, 6 (2009), 1731-1768.

[12] G. Dwivedi And J. Tyagi, Remarks on the qualitative questions for biharmonic operators, Taiwanese J. Math 19, 6 (2015), 1743-1758.

[13] G. DwiVedi AND J. Tyagi, Picone's identity for biharmonic operators on Heisenberg group and its applications, NoDEA Nonlinear Differential Equations Appl. 23, 2 (2016), 1-26.

[14] Y. Fu, V. D. RĂDUlescu AND B. ZHANG, Hodge decomposition of variable exponent spaces of Clifford-valued functions and applications to Dirac and Stokes equations, Comput. Math. Appl. 70, 4 (2015), 691-704.

[15] X. Fan, Q. Zhang And D. Zhao, Eigenvalues of $p(x)$-Laplacian Dirichlet problem, J. Math. Anal. Appl. 302, 2 (2005), 306-317. 
[16] P. HaRjulehto, P. HÄStÖ AND Ú. V. LÊ, ET AL., Overview of differential equations with nonstandard growth, Nonlinear Anal. 72, 12 (2010), 4551-4574.

[17] S. LEvine (Eds), An adaptive variational model for image decomposition, Energy Minimization Methods in Computer Vision and Pattern Recognition, Springer-Verlag, Berlin, 2005.

[18] M. MihăILESCU AND C. VARGA, Multiplicity results for some elliptic problems with nonlinear boundary conditions involving variable exponents, Comput. Math. Appl. 62, 9 (2011), 3464-3471.

[19] P. NiU, H. ZHANG AND Y. WANG, Hardy type and Rellich type inequalities on the Heisenberg group, Proc. Amer. Math. Soc. 129, 12 (2001), 3623-3630.

[20] M. Picone, Sui valori eccezionali di un parametro da cui dipende un'equazione differenziale lineare ordinaria del second'ordine, Ann. Scuola Norm. Sup. Pisa CI. Sci. 11, 1(1910), 1-144.

[21] M. RŮŽIČKA, Electrorheological Fluids: Modeling and Mathematical Theory, Springer-Verlag, Berlin, 2000.

[22] K. R. RAJAGOPAL AND M. RŮŽIČKA, Mathematical modeling of electrorheological materials, Cont. Mech. Therm. 13, 1(2001), 59-78.

[23] J. TIIROLA, Image decompositions using spaces of variable smoothness and integrability, SIAM J. Imaging Sci. 7, 3 (2014), 1558-1587.

[24] J. TYAGI, A nonlinear Picone's identity and its applications, Appl. Math. Lett. 26, 6 (2013), 624-626.

[25] N. Yoshida, Picone identity for quasilinear elliptic equations with $p(x)$-Laplacians and Sturmianian comparison theory, Appl. Math. Comput. 225, 1 (2013), 79-91.

[26] V. V. Zhikov, Averaging of functionals of the calculus of variations and elasticity theory, Math. USSR. Izv. 29, 1 (1987), 33-36.

[27] Q. ZHANG, Existence and asymptotic behavior of positive solutions for variable exponent elliptic systems, Nonlinear Anal. 70, 1 (2009), 305-316. 